\title{
Magnetic resonance imaging evaluation of retro-bulbar optic nerve in patients with optic disc pit maculopathy
}

\author{
Katarzyna Baltaziak', Lucyna Baltaziak', Rashed Mustafa Nazzal $\odot^{2}$, Robert Rejdak ${ }^{2}$, Tomasz Pikuła ${ }^{3}$ \\ ${ }^{1}$ Medical Students Ophthalmology Interest Group, General Ophthalmology Clinic, Medical University of Lublin, Lublin, Poland \\ ${ }^{2}$ Department of General Ophthalmology, Medical University of Lublin, Lublin, Poland \\ ${ }^{3}$ Department of Interventional Radiology and Diagnostic Imaging, Medical University of Lublin, Poland
}

\begin{abstract}
BACKGROUND: Optic disc pit (ODP) is a congenital anomaly characterized by indented area of the optic nerve head most likely originating from the incomplete closure of the superior edge of the embryonic fissure. Maculopathy can occasionally complicate this anomaly as intra-retinal and sub-retinal fluid at the macula. In result, maculopathy is associated with a poor visual prognosis due to a serous macular detachment, formation of macular hole, or atrophy of the retinal pigmented epithelium. In this case series, we report of three patients with unilateral ODP.

CASE REPORT: We attempted to analyze the morphologic changes seen in the ODP and evaluate patients with a complete ophthalmologic evaluation, fundus color photography, spectral-domain optical coherence tomography (SD-OCT) scanning, and magnetic resonance imaging (MRI) of globe and orbit. Magnetic resonance imaging scan was done for optic disc diameter (ODD) and optic nerve sheath diameter (ONSD).

CONCLUSION: We noticed that in the patient who presented with neurosensory detachment in the macula, the ONSD is larger than in the fellow eye and is larger than the remaining two patients with ODP and without neurosensory detachment of the retina.
\end{abstract}

KEY WORDS: optic disc pit; OCT; MRI; maculopathy; optic nerve

Ophthalmol J 2020; Vol. 5, 25-28

\section{INTRODUCTION}

Optic disc pit (ODP) is a rare inherited anomaly of the head of the optic nerve. It typically appears in one eye (although 15\% are bilateral). Optic disc pit presents as oval, hypo-pigmented discolored scooping of the optic disc, most frequentlyin the temporal or inferio-temporal segment of the optic disc. At times, it can be found in other sector of the optic nerve [1]. The incidence of ODP is 1 in 10,000 people without gender predilection [2]. Optic disc pit is usually asymptomatic, but if large enough, it can be associated with visual field defects. In the absence of associated maculopathy, vision is typically unaffected $[1,2]$.

Regarding the origin of the fluid that causes the maculopathy, the exact mechanism remains unclear and several competing theories have suggested it might originate from liquified vitreous humor between the retina is the vitrous membrane, leakage of serum from blood vessels at the base of the pit or leakage of the cerebrospinal fluid (CSF) from the choroid [3-5].

The aim of this study was to review the ODP case series including the natural history of the disease to 

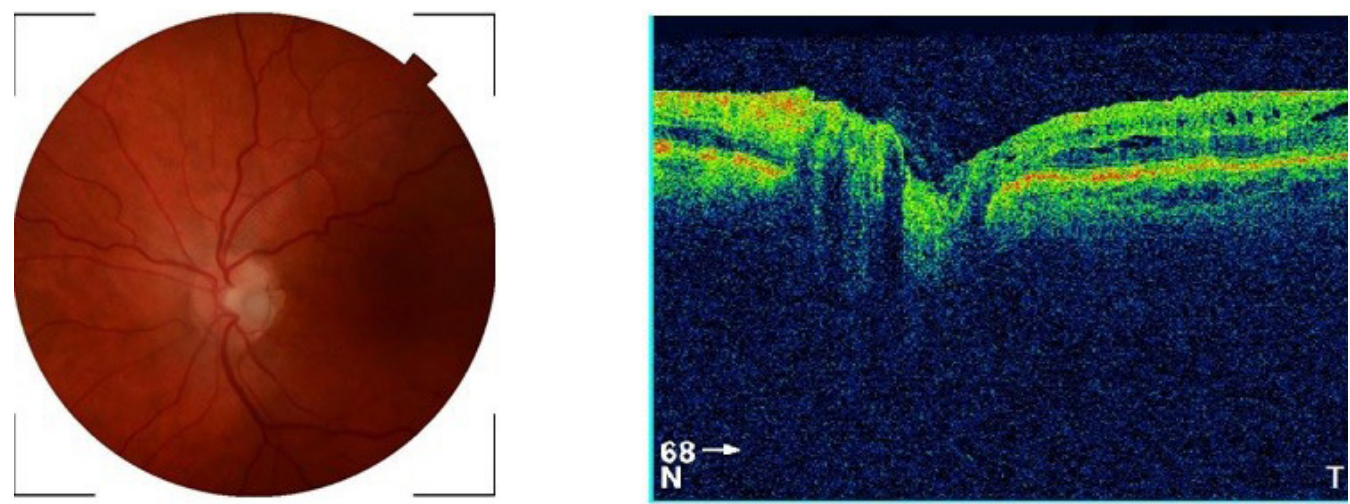

FIGURE 1. Coloured fundus photography and optical coherence tomography (OCT) of the optic disco of left eye for the Case 1

gain better understanding of clinical and pathological circumstances involved in development of the ODP-related maculopathy, and at least in part to develop insights into the prediction of when and if ODP patients may develop maculopathy.

\section{CASE REPORTS}

We attempted to analyze the morphologic changes seen in the ODP and evaluate patients with a complete ophthalmologic evaluation, fundus color photography, spectral-domain optical coherence tomography (SD-OCT) scanning, and Magnetic resonance imaging (MRI) of globe and orbit. MRI scan was done for optic disc diameter (ODD) and optic nerve sheath diameter (ONSD).

\section{CASE 1}

A 70-year-old woman with chief complaint of gradual worsening of vision over the last few months. On examination, she was found to have a best corrected visual acuity of 0.4 . Fundus exami- nation showed optic disc pit complicated by maculopathy which was confirmed by OCT (Fig. 1). MRI scan was done for optic disc diameter (ODD) and optic nerve sheath diameter (ONSD) and both measurements were within normal limits (Fig. 4A).

\section{CASE 2}

A 42-year-old man presented to our clinic for check-up. The visual acuity was 1.0 and the fundus examination showed an incidental finding of ODP in his left eye as illustrated in the colored fundus photograph and OCT (Fig. 2). And MRI scan was obtained also and measurements for ODD and ONSD were within normal limits (Fig. 4B).

\section{CASE 3}

A 41-year-old woman, presented with gradual worsening of vision in left eye. The visual acuity was 0.1 in the left eye. Fundus exam showed macular neurosensory detachment confirmed by OCT, and associated optic nerve pit; there was also areas of RPE pigmentary changes (Fig. 3). To measure the
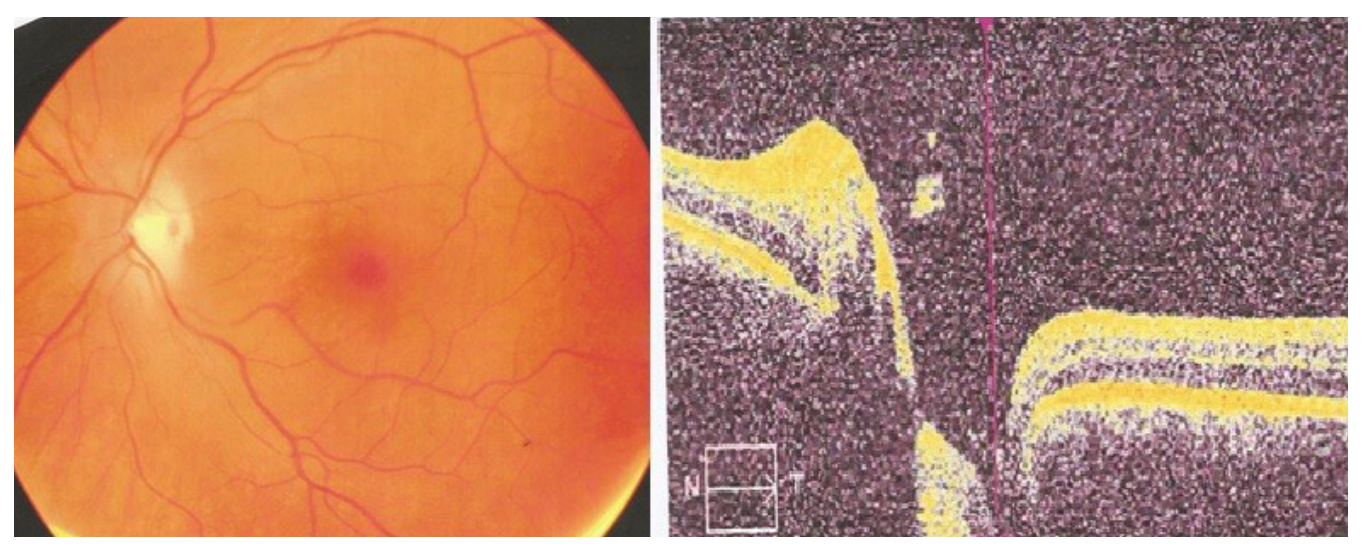

FIGURE 2. Coloured fundus photography and optical coherence tomography (OCT) of the optic disco of left eye for the Case 2 

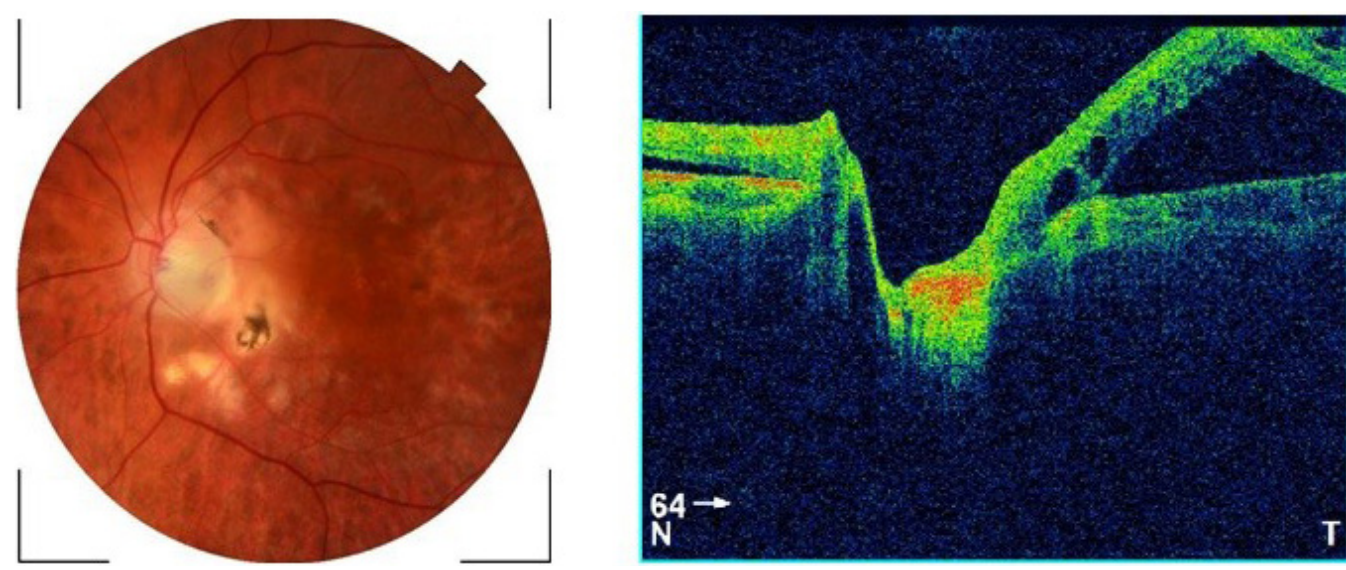

FIGURE 3. Coloured fundus photography and optical coherence tomography (OCT) of the optic disco of left eye for the Case 3

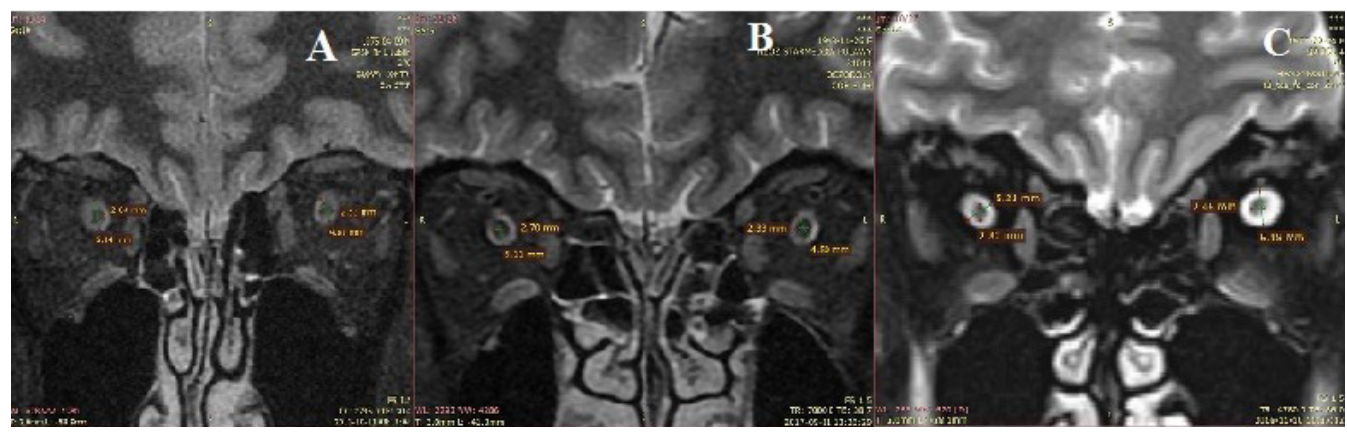

FIGURE 4. Constructive interference in steady state T2-weighted sequences imaging (CISS MRI) for the three patients. A. Case 1; B. Case 2; C. Case 3

\begin{tabular}{|l|c|c|c|c|c|}
\hline \multicolumn{2}{|l|}{ Table 1. Demographics of the patients } \\
\hline Patient & Eye & Gender & Visual acuity & Symptoms & Location of the ONP \\
\hline 1 & Left & Female & 0.4 & Bluring of vision & Temporal \\
\hline 2 & Left & Male & 1.0 & Asymptomatic & Inferiotemporal \\
\hline 3 & Left & Female & 0.1 & Decreased of vision & Inferiotemporal \\
\hline
\end{tabular}

OPN — optic nerve pit

size of the ONSD we used MRI scans targeting the retro-bulbar part of the optic nerve. Based on the radiologist recommendation we used a special protocol called constructive interference in steady state T2-weighted sequences (CISS MRI), which has demonstrated the increase in 3D dimensions of abnormally swollen ONSD on the CISS MRI (Fig. 4C).

Summary of patient demographics is shown in Table 1.

\section{DISCUSSION}

It has been always known that maculopathy is a well-recognized complication of optic disc pit, which usually results in poor visual outcome [2]. In this case series of three patients, we evaluated the patients with full ophthalmological examination in addition to MRI scan for the retro-bulbar part of the optic nerve.

Geeraerts et al. showed that the mean ONSD in heal thy people was $(5.08 \pm 0.52 \mathrm{~mm})$, while the normal ODD in healthy people was $(2.70 \pm 0.23 \mathrm{~mm}$; $\mathrm{p}=0.26)[6]$.

In our cases, we compared the diameter of the ODD in both eyes of the same patient, and between the three patients and they were all within normal limits. Then, we measured the ONSD that surrounds the retro-bulbar part of the optic nerve 
Table 2. Summary of measurements by magnetic resonance imagong (MRI)

\begin{tabular}{|l|c|c|c|c|}
\hline \multirow{2}{*}{ Patient } & \multicolumn{2}{|c|}{ OND [mm] } & \multicolumn{2}{c|}{ ONSD [mm] } \\
\cline { 2 - 5 } & Right eye & Left eye & Right eye & 4.6 \\
\hline 1 & 2.6 & 2.5 & 5.1 & 4.8 \\
\hline 3 & 2.7 & 2.3 & 5.1 & 6.2 \\
\hline
\end{tabular}

OND — optic nerve diameter; ONSD — optic nerve sheath diameter

between both eyes of the same patient and among the three patients (Tab. 2). In this case series, we used the other eye as a control group. We noticed that in the third patient who presented with neurosensory detachment in the macula, the ONSD is larger in the left eye than the left eye and is larger than all three patients. This was better illustrated by doing a modified protocol in the MRI scan known as CISS - is a fully refocused fast-gradient echo sequence that is mainly used in the assessment of the central nervous system structure and lesions. Advantages of steady-state imaging are short acquisition times, high signal-to-noise ratio, and better contrast-to-noise ratio. In addition, a cisternographic effect of CISS allows assessments of cranial nerves which was relevant in this study [7].

Whether this result is of clinical significance or not, needs further study. We are aware of the very small sample size in this report. But such an association has never been reported before, hence further studies might be needed to reach a clinical significance.

Medical management of ODP maculopathy remains challenging although there are reports of spontaneous resolution of the disease. Nevertheless, the current management of the ODP maculopathy involves several surgical approaches and presents possibility of postsurgical complications for affected patients undergoing vitrectomy due to the ODP maculopathy. Alternatives to vitrectomy include either gas tamponade or laser photocoagulation.
Other less frequently performed options including scleral buckling and inner fenestration, have been also reported [8].

\section{Acknowledgements}

None.

\section{Conflict of interests}

None.

\section{REFERENCES}

1. Wiethe T. Ein Fall von angelborener Difformitaet der Sehnerven-papille. Arch Augenheilkd. 1882; 11: 14-19.

2. Gass JD. Serous detachment of the macula. Secondary to congenital pit of the optic nervehead. Am J Ophthalmol. 1969; 67(6): 821-841, doi: 10.1016/0002-9394(69)90075-0, indexed in Pubmed: 5795739.

3. Irvine AR, Crawford JB, Sullivan $\mathrm{JH}$, et al. The pathogenesis of retinal detachment with morning glory disc and optic pit. Trans Am Ophthalmol Soc. 1986; 84(3): 280-292, indexed in Pubmed: 3590472.

4. Gowdar JP, Rajesh B, Giridhar A, et al. An insight into the pathogenesis of optic disc pit-associated maculopathy with enhanced depth imaging. JAMA Ophthalmol. 2015; 133(4): 466-469, doi: 10.1001/ jamaophthalmol.2014.6093, indexed in Pubmed: 25675311.

5. Moisseiev E, Moisseiev J, Loewenstein A. Optic disc pit maculopathy: when and how to treat? A review of the pathogenesis and treatment options. Int J Retina Vitreous. 2015; 1: 13, doi: 10.1186/s40942-0150013-8, indexed in Pubmed: 27847606.

6. Geeraerts T, Newcombe VFJ, Coles JP, et al. Use of T2-weighted magnetic resonance imaging of the optic nerve sheath to detect raised intracranial pressure. Crit Care. 2008; 12(5): R114, doi: 10.1186/ cc7006, indexed in Pubmed: 18786243.

7. Gonçalves F, Amaral L. Constructive Interference in Steady State Imaging in the Central Nervous System. Eur Neurol Rev. 2011; 6(2): 138, doi: 10.17925/enr.2011.06.02.138.

8. Chatziralli I, Theodossiadis P, Theodossiadis GP. Optic disk pit maculopathy: current management strategies. Clin Ophthalmol. 2018; 12: 1417-1422, doi: 10.2147/0PTH.S153711, indexed in Pubmed: 30127591. 